\title{
Sample Extractor for Serial Crystallography at XFELs and Synchrotron sources
}

\author{
Irimpan Mathews $^{1}$, Aina Cohen ${ }^{1}$, Artem Lyubimov ${ }^{1}$, Michael Soltis ${ }^{1}$ \\ ${ }^{1}$ Stanford University/SLAC, Menlo Park, United States \\ E-mail: iimathew@slac.stanford.edu
}

The short X-ray pulses produced by X-ray Free Electron Lasers (XFELs) can produce diffraction information on time scales that outrun radiation damage processes, enabling structural investigations using extremely small and weakly diffracting crystals at ambient temperatures. Since each crystal exposed is destroyed in the process, full datasets are assembled from a series of still diffraction images collected from many individual crystals delivered in progression, a process known as Serial Femtosecond Crystallography (SFX). Unfortunately, the technical challenges and complexity of sample delivery using crystal injectors often limits the wider use of SFX.

Here we describe a new sample delivery device for SFX, the Sample Extractor, that may be used to deliver delicate crystals directly from native crystallization solutions. The Sample Extractor operates at ambient temperatures and pressures minimizing the mechanical stress subjected to the crystals. Furthermore, operation is not sensitive to crystal size. The device was successfully used to collect complete SFX datasets at the LCLS XFEL and the SSRL synchrotron. We noticed significant differences especially in the active site between the room temperature XFEL structure and the same structure at $100 \mathrm{~K}$. Details of the device including fabrication, operation, data collection and recent results will be presented.

Keywords: XFEL, Serial Crystallography, Sample Delivery 